\title{
ON APPROXIMATION BY WALSH FUNCTIONS
}

\section{SHIGEKI YANO}

Let $\phi_{n}(x), n=0,1,2, \cdots$, be the Rademacher functions, that is,

$$
\begin{aligned}
& \phi_{0}(x)=1 \quad(0 \leqq x<1 / 2), \quad \phi_{0}(x)=-1 \quad(1 / 2 \leqq x<1), \\
& \phi_{0}(x+1)=\phi_{0}(x), \quad \phi_{n}(x)=\phi_{0}\left(2^{n} x\right) \quad(n=1,2, \cdots) .
\end{aligned}
$$

Then the Walsh functions are defined by

$$
\psi_{0}(x) \equiv 1, \quad \psi_{n}(x)=\phi_{n_{1}}(x) \phi_{n_{2}}(x) \cdots \phi_{n_{r}}(x)
$$

for $n=2^{n_{1}}+2^{n_{2}}+\cdots+2^{n_{r}}$, where the integers $r_{i}$ are uniquely determined by $n_{i+1}<n_{i}$. As is well known, the Walsh functions $\left\{\psi_{n}(x)\right\}$ form a complete orthonormal set. Every periodic function integrable on $(0,1)$ will have associated with it a Walsh-Fourier series

$$
f(x) \sim \sum_{n=0}^{\infty} c_{n} \psi_{n}(x)
$$

where the coefficients are given by

$$
c_{n}=\int_{0}^{1} f(x) \psi_{n}(x) d x \quad(n=0,1,2, \cdots) .
$$

Recently N. J. Fine ${ }^{1}$ has proposed the following problem: if $f(x) \in \operatorname{Lip} \alpha, 0<\alpha<1,2$ and if we denote the arithmetic mean of the Walsh-Fourier series of $f(x)$ by $\sigma_{n}(x ; f)$, then $\sigma_{n}(x ; f)-f(x)=O\left(n^{-\alpha}\right)$ ? We shall give here an affirmative answer of this problem and its generalization. For the definitions, notations, and fundamental results on the Walsh functions used here we shall refer to Fine's paper.

TheOREM 1. If $f(x) \in \operatorname{Lip} \alpha, 0<\alpha<1$, then

$$
\sigma_{n}(x ; f)-f(x)=O\left(n^{-\alpha}\right) .
$$

This theorem can be proved by the following lemmas.

LEMMA $10^{3}$ Let $K_{n}(t)$ be the Fejer kernel for the Walsh functions, and let $I_{p}^{(n)}$ denote the interval $p \cdot 2^{-n} \leqq t<(p+1) 2^{-n}, 0 \leqq p<2^{n}$. Then for $n \geqq 2$,

Received by the editors July 10, 1950 and, in revised form, October 9, 1950 and November 13, 1950.

${ }^{1}$ On the Walsh functions, Trans. Amer. Math. Soc. vol. 65 (1949) pp. 372-414.

2 Of course, the periodicity with period 1 is assumed.

8 The author wishes to thank the referee for his valuable suggestions for the proof of this lemma. 
(6)
(i) $K_{2^{n}}(t)=\frac{2^{n}+1}{2}$
$\left(t \in I_{0}^{(n)}\right)$
(ii) $K_{2^{n}}(t)=2^{n-r-2}$
$\left(t \in I_{2^{r}}^{(n)}, r=0,1, \cdots, n-1\right)$
(iii) $K_{2^{n}}(t)=0$
elsewhere in $(0,1)$.

Proof. It is obvious that (6) holds for $n=2$. Suppose that (6) holds for an $n \geqq 2$. Then we use the identity (cf. Fine, Lemma 3 )

$$
K_{2^{n+1}}(t)=\frac{1+\psi_{2^{n}}(t)}{2} K_{2^{n}}(t)+\frac{1}{2} D_{2^{n}}(t)
$$

to prove that it holds for $n+1$. We know that $K_{2^{n+1}}(t)$ is constant on $I_{p}^{(n+1)}, 0 \leqq p<2^{n+1}$. On $I_{0}^{(n+1)}$ and $I_{1}^{(n+1)}, D_{2^{n}}(t)=2^{n}$; on $I_{2 m}^{(n+1)}, \psi_{2^{n}}(t)=1$, and on $I_{2 m+1}^{(n+1)}, \psi_{2^{n}}(t)=-1$. Hence on $I_{0}^{(n+1)}, K_{2^{n+1}}(t)=K_{2^{n}}(t)+2^{n-1}$ $=\left(2^{n+1} / 2+2^{n-1}=\left(2^{n+1}+1\right) / 2\right.$, so that (i) is true for $n+1$. On $I_{1}^{(n+1)}$, $K_{2^{n+1}}(t)=2^{n-1}$, so that (ii) is true for $n+1$, with $r=0$. For $2^{-n} \leqq t<1$, $D_{2^{n}}(t)=0$; hence if $t \in I_{2^{r}}^{(n+1)} \subset I_{2^{r-1}}^{(n)}$, for $1 \leqq r<n+1$, we have

$$
K_{2^{n+1}}(t)=K_{2^{n}}(t)=2^{n-(r-1)-2}=2^{(n+1)-r-2},
$$

and (ii) is true for $0 \leqq r<n+1$. Now (iii) follows from the facts that the integral of $K_{2^{n+1}}(t)$ over $(0,1)$ is 1 , that $K_{2^{n+1}}(t)$ is non-negative, and that the integral over the interval specified in (i) and (ii) (with $n$ replaced by $n+1$ ) is

$$
2^{-(n+1)}\left\{\frac{2^{n+1}+1}{2}+2^{n-1}+\cdots+1+\frac{1}{2}\right\}=1 .
$$

This completes the proof of the lemma.

LEMMA 2. Under the assumption of Theorem 1,

$$
\sigma_{2^{n}}(x ; f)-f(x)=O\left(2^{-\alpha n}\right) .
$$

Proof. We may write

$$
\sigma_{2^{n}}(x ; f)-f(x)=\int_{0}^{1}[f(x+t)-f(x)] K_{2^{n}}(t) d t .
$$

Then by Lemma 1,

$$
\begin{aligned}
\sigma_{2^{n}}(x ; f)-f(x)= & \frac{2^{n}+1}{2} \int_{0}^{2-n}[f(x+t)-f(x)] d t \\
& +\sum_{i=0}^{n-1} 2^{n-i-2} \int_{2^{-n+i}}^{2-n+i+2-n}[f(x+t)-f(x)] d t
\end{aligned}
$$


By virtue of the fact that $|(x+t)-x| \leqq t, 0 \leqq x<1,0 \leqq t<1$ (cf. Fine, \$2.(2.8)), and $0<\alpha<1$,

$$
\begin{aligned}
\sigma_{2^{n}}(x ; f)-f(x) & =2^{n} \int_{0}^{2-n} O\left(t^{\alpha}\right) d t+\sum_{i=0}^{n-1} 2^{n-i-2} \int_{2^{-n+i}}^{2-n+i+2-n} O\left(t^{\alpha}\right) d t \\
& =O\left(2^{-\alpha n}\right)+\sum_{i=0}^{n-1} 2^{n-i-2} O\left(2^{\alpha(-n+i+1)}\right) 2^{-n} \\
& =O\left(2^{-\alpha n}\right)+O\left(2^{-\alpha n}\right) \sum_{i=0}^{n-1} 2^{-(1-\alpha) i} \\
& =O\left(2^{-\alpha n}\right)
\end{aligned}
$$

and this proves the lemma.

We shall now prove Theorem 1. Let $n=2^{n_{1}}+2^{n_{2}}+\cdots+2^{n_{r}}$, $n_{1}>n_{2}>\cdots>n_{r} \geqq 0$, and $n^{\prime}=n-2^{n_{1}}, n^{(i)}=n^{(i-1)}-2^{n_{i}}, i=2,3, \cdots$, $r$. Then it is known (Fine, Lemma 4) that

$$
n K_{n}(t)=\sum_{i=1}^{r} 2^{n_{i} \psi_{n-n^{(i)}}}(t) K_{2^{n_{i}}}(t)+\sum_{i=1}^{r} n^{(i)} D_{2^{n_{i}}}(t) .
$$

Therefore we may write

$$
\begin{aligned}
\sigma_{n}(x ; f)-f(x)= & \int_{0}^{1}[f(x+t)-f(x)] K_{n}(t) d t \\
= & \frac{1}{n} \sum_{i=1}^{r} 2^{n_{i}} \int_{0}^{1}[f(x+t)-f(x)] \psi_{n-n^{(i)}}(t) K_{2^{n_{i}}}(t) d t \\
& +\frac{1}{n} \sum_{i=1}^{r} n^{(i)} \int_{0}^{1}[f(x+t)-f(x)] D_{2^{n_{i}}}(t) d t \\
= & P_{n}+Q_{n}
\end{aligned}
$$

say. By Lemma 2,

$$
\begin{aligned}
\left|P_{n}\right| & \leqq \frac{1}{n} \sum_{i=1}^{r} 2^{n_{i}} \int_{0}^{1}|f(x+t)-f(x)| K_{2^{n_{i}}(t) d t} \\
& =\frac{1}{n} O\left(\sum_{i=1}^{r} 2^{n_{i}} 2^{-\alpha n_{i}}\right)=\frac{1}{n} O\left(\sum_{i=1}^{n_{1}} 2^{(1-\alpha) i}\right) \\
& =\frac{1}{n} O\left(2^{(1-\alpha) n_{1}}\right)=O\left(n^{-\alpha}\right) .
\end{aligned}
$$

On the other hand, by the property of $D_{2^{n_{i}}(t)}$ (already used in the proof of Lemma 1), 


$$
\left|\int_{0}^{1}[f(x+t)-f(x)] D_{2^{n_{i}}}(t) d t\right| \leqq 2^{n_{i}} \int_{0}^{1} O\left(t^{\alpha}\right) d t=O\left(2^{-\alpha n_{i}}\right) .
$$

By definition, $n^{(i)}<2^{n_{i}}$. Therefore

$$
\left|Q_{n}\right| \leqq \frac{1}{n} O\left(\sum_{i=1}^{r} n^{(i)} 2^{-\alpha n_{i}}\right)=\frac{1}{n} O\left(\sum_{i=1}^{r} 2^{(1-\alpha) n_{i}}\right)=O\left(n^{-\alpha}\right) .
$$

(12), (13), and (15) prove the theorem.

More generally we can prove the following theorem.

Theorem 2. If $f(x) \in \operatorname{Lip} \alpha, 0<\alpha<1$, then for any $\beta>\alpha$

$$
\sigma_{n}^{(\beta)}(x ; f)-f(x)=O\left(n^{-\alpha}\right),
$$

where $\sigma_{n}^{(\beta)}(x ; f)$ denotes the $(C, \beta)$ mean of the Walsh-Fourier series of $f(x)$, that is,

$$
\begin{aligned}
\sigma_{n}^{(\beta)}(x ; f) & =\frac{1}{A_{n-1}^{(\beta)}} \sum_{k=0}^{n-1} A_{n-k-1}^{(\beta)} c_{k} \psi_{k}(x), \\
A_{n}^{(\beta)} & =\frac{(\beta+1)(\beta+2) \cdots(\beta+n)}{n !}, A_{0}^{(\beta)}=1 .
\end{aligned}
$$

Proof. Let $K_{n}^{(\beta)}(t)$ be the $(C, \beta)$-kernel for the Walsh functions and let $n=2^{n_{1}}+2^{n_{3}}+\cdots+2^{n_{r}}, n_{1}>n_{2}>\cdots>n_{r} \geqq 0$. Then, using the notations in the proof of Theorem 1, we may write

$$
\begin{aligned}
A_{n-1}^{(\beta)} K_{n}^{(\beta)}(t) & =\sum_{k=0}^{n-1} A_{n-k-1}^{(\beta)} \psi_{k}(t) \\
= & {\left[\sum_{k=0}^{2 n_{1}-1}+\sum_{k=2^{n_{1}}}^{2 n_{1}+2 n_{2}-1}+\cdots+\sum_{k=2^{n_{1}} \cdots+2^{n_{r-1}}}^{n-1}\right] A_{n-k-1}^{(\beta)} \psi_{k}(t) . }
\end{aligned}
$$

Since $\psi_{2^{n_{i}-j-1}}(t)=\psi_{j}(t) \psi_{2^{n_{i}-1}}(t)$ for $0 \leqq j<2^{n_{i}}$, it follows that

$$
\begin{aligned}
& \sum_{k=2^{n_{1}}+2^{n_{2}}+\cdots+2^{n_{i-1}}}^{2 n_{1}+2 m_{+}+\cdots+2 n_{i}-1} A_{n-k-1}^{(\beta)} \psi_{k}(t) \\
& =\psi_{2^{n_{1}}+2^{n_{2}}+\cdots+2^{n_{i-1}}}(t) \sum_{j=0}^{2 n_{i}-1} A_{n^{(i)}+j}^{(\beta)} \psi_{2^{n_{i-j-1}}}(t) \\
& =\psi_{2^{n_{1}}+\cdots+2^{n_{i}-1}}(t) \sum_{k=0}^{2 n_{i}-1} A_{n^{(i)}+k}^{(\beta)} \psi_{k}(t) \\
& =\psi_{n-n^{(i)}-1}(t) \sum_{k=0}^{2 n_{i}-1} A_{n^{(i)}+k}^{(\boldsymbol{\beta})} \psi_{k}(t) .
\end{aligned}
$$


Substituting (18) into (17), we get

$$
A_{n-1}^{(\beta)} K_{n}^{(\beta)}(t)=\sum_{i=1}^{r} \psi_{n-n^{(i)}-1}(t) \sum_{k=0}^{2 n_{i}-1} A_{n^{(i)}+k}^{(\beta)} \psi_{k}(t) .
$$

Applying Abel's transformation twice, we get

$$
\begin{aligned}
& A_{n-1}^{(\beta)} K_{n}^{(\beta)}(t)=\sum_{i=1}^{r} \psi_{n-n^{(i)}-1}(t)\left[\sum_{k=0}^{2 n_{i}-3} k A_{n^{(i)}+k}^{(\beta-2)} \cdot K_{k+1}(t)\right. \\
& \left.+\left(2^{n_{i}}-2\right) A_{n^{(i-1)}-2}^{(\beta-1)} K_{2^{n_{i-1}}}(t)+A_{n^{(i-1)-1}}^{(\beta)} D_{2^{n_{i}}}(t)\right] .
\end{aligned}
$$

Therefore

$$
\begin{aligned}
& \left|\sigma_{n}^{(\beta)}(x ; f)-f(x)\right| \\
& \leqq \frac{1}{A_{n-1}^{(\beta)}} \sum_{i=1}^{r}\left[\sum_{k=1}^{2 n_{i}-3} k\left|A_{n}^{(\beta)+k}\right| \int_{0}^{1}|f(x+t)-f(x)|\left|K_{k+1}^{(\beta)}(t)\right| d t\right. \\
& +2^{n_{i}} A_{n^{(i-1)}-2}^{(\beta-1)} \int_{0}^{1}|f(x+t)-f(x)|\left|K_{2^{n_{i}-1}}(t)\right| d t \\
& +A_{n^{(i-1)}-1}^{(\beta)} \int_{0}^{1}|f(x+t)-f(x)| D_{2^{n s}}(t) d t \\
& =\frac{1}{A_{n-1}^{(\beta)}} \sum_{i=1}^{r}\left(P_{i}+Q_{i}+R_{i}\right),
\end{aligned}
$$

say. By Theorem 1

$$
\begin{aligned}
P_{i} & =\sum_{k=1}^{2 n_{i}-3} k\left|A_{n^{(i)}+k}^{(\beta-2)}\right| \int_{0}^{1}|f(x+t)-f(x)|\left|K_{k+1}(t)\right| d t \\
& =O\left(\sum_{k=1}^{2 n_{i}} k \cdot k^{\beta-2} \cdot K^{-\alpha}\right)=O\left(2^{(\beta-\alpha) n_{i}}\right), \quad A_{n}^{(i)+k+1} \rightarrow A_{n}^{(i)}+k j
\end{aligned}
$$

where we assume that $\alpha<\beta<1$ and use the fact that $A_{n}^{(\beta-2)+k+}$ $=O\left(k^{\beta-2}\right)$. It is obvious that

$$
Q_{i}=O\left(2^{n_{i}(\beta-\alpha)}\right), \quad R_{i}=\left(2^{n_{i}(\beta-\alpha)}\right) .
$$

Thus by (19), (20), (21) and by the assumption $\beta>\alpha$, we have

$$
\begin{aligned}
\sigma_{n}^{(\beta)}(x ; f)-f(x) & =O\left(n^{-\beta} \sum_{i=1}^{r} 2^{n_{i}(\beta-\alpha)}\right)=O\left(n^{-\beta} \sum_{i=1}^{n_{1}} 2^{i(\beta-\alpha)}\right) \\
& =O\left(n^{-\beta} n^{(\beta-\alpha)}\right)=O\left(n^{-\alpha}\right),
\end{aligned}
$$

and this completes the proof of Theorem 2 . 
Theorems 1 and 2 have their analogues in the case of integrated Lipschitz condition; that is, the following theorem holds:

Theorem 3. If $f(x) \in \operatorname{Lip}(\alpha, p), 0<\alpha<1, p \geqq 1$, then for any $\beta>\alpha$

$$
\left(\int_{0}^{1}\left|\sigma_{n}^{(\beta)}(x ; f)-f(x)\right|^{p} d x\right)^{1 / p}=O\left(n^{-\alpha}\right) .
$$

Since the proof is analogous to the preceding one, we shall omit it.

TôHoKU UNIVERSITY

\section{POSITIVE INFINITIES OF POTENTIALS}

\section{WALTER RUDIN}

Let $R$ denote Euclidean 3-space. The following theorem is due to Evans [1, p. 421]. ${ }^{1}$

Let $E$ be a closed bounded set of capacity zero in $R$. There exists a distribution of positive mass $\mu(e)$ entirely on $E$, such that its potential $V(M)=\int_{R}(1 / M P) d \mu(P)$ is infinite at every point of $E$ and at no other points.

A proof of the two-dimensional analogue was published by Noshiro [2]. In the present note we show, by a modification of Evans' construction, that an absolutely continuous distribution exists whose potential is infinite on the preassigned set $E$ only. More precisely, our result, extended to unbounded sets, is as follows:

Theorem. Let $E$ be a closed set of capacity zero in $R$, and let $G$ be an open set containing $E$. Then there exists a non-negative function $f$ which is summable on $R$, such that the superharmonic function (that is, the potential)

$$
F(M)=\int_{R} \frac{1}{M P} f(P) d P
$$

is infinite on $E$, is continuous in $R-E$, and is harmonic in $R-\bar{G}$. ( $\bar{G}$ denotes the closure of $G$.)

Analogous results evidently hold in two and in more than three dimensions.

Presented to the Society, February 24, 1951; received by the editors December 4, 1950.

${ }^{1}$ Numbers in brackets refer to the references at the end of the paper. 